\title{
Proyecto cultural para la adquisición de competencias laborales en Ingeniería Industrial
}

\section{Cultural project for the acquisition of labor skills in Industrial Engineering}

JIMÉNEZ-HIDALGO, Guadalupe †**, MONTOYA-PONCE, Javier, MARTINEZ-ACOSTA, María Teresa y PEREA-SEAÑEZ, Loreley

\author{
Tecnológico Nacional de México / Instituto Tecnológico de Ciudad Jiménez. \\ ID $1^{\mathrm{er}}$ Coautor: Javier, Montoya-Ponce / ORC ID: 0000-0002-9857-7961 \\ ID $2^{\text {do }}$ Coautor: María Teresa, Martínez-Acosta / ORC ID: 0000-0001-8934-4843 \\ ID $3^{\text {er }}$ Coautor: Loreley, Perea-Seañez / ORC ID: 0000-0002-3391-383X
}

ID $1^{\text {er }}$ Autor: Guadalupe, Jiménez-Hidalgo / ORC ID: 0000-0002-8257-3093, CVU CONACYT ID: 1013757

DOI: $10.35429 / J P D .2019 .8 .3 .35 .40$

Recibido 01 de Abril, 2019; Aceptado 30 de Junio, 2019

\begin{abstract}
Resumen
La formación integral en la educación superior permite el desarrollo pleno de las potencialidades del ser humano; adicionalmente de lo académico, se debe cultivar el crecimiento personal, entre ellos la cultura y el cuidado del medio ambiente. Por lo que los docentes deben concebir actividades de aprendizaje que promuevan este desarrollo.

Se presenta la experiencia de una intervención educativa que promueve la generación de competencias laborales utilizando un proyecto cultural, entrelazando las materias de economía, desarrollo sustentable y metrología y normalización, realizada a partir de una investigación sobre los estilos de aprendizaje de los estudiantes de educación superior de Ingeniería industrial los cuales presentan una preferencia al estilo teórico, seguido de activo y pragmático, por lo que se realizó una estrategia didáctica que promoviera el cuidado de medio ambiente, las tradiciones del día de muertos, el trabajo en equipo, los análisis de costos, la medición de las estructuras vinculando los aprendizajes teóricos, prácticos y actitudinales, con la producción de una Calaca a partir del reuso de material que tuviera proporciones óseas acordes a sus dimensiones. La participación de los estudiantes en el evento cultural con sus propuestas de Catrinas mostró sus competencias de las asignaturas.
\end{abstract}

Competencia laboral, estrategias didácticas, educación superior

\begin{abstract}
Integral training in university degree allows the full development of the potential of the human being; In addition to academics, personal growth must be cultivated, including culture and environmental care. So teachers should conceive learning activities that promote this development. The experience of an educational intervention that promotes the generation of labor competencies using a cultural project, intertwining the subjects of economics, sustainable development and metrology and standardization, based on investigation into the learning styles of university students of Industrial engineering, they presents a preference to the theoretical style, followed by active and pragmatic, so a didactic strategy was carried out that promoted the care of the environment, the traditions of the day of the dead, teamwork, cost analysis, the measurement of the structures linking the theoretical, practical and attitudinal learning, with the production of a Calaca whit reuse material that had bone proportions according to its dimensions. The participation of the students in the cultural event with their proposals of Catrinas showed their competences of the subjects.
\end{abstract}

Labor competence, teaching strategies, higher education

Citación: JIMÉNEZ-HIDALGO, Guadalupe, MONTOYA-PONCE, Javier, MARTINEZ-ACOSTA, María Teresa y PEREA-SEAÑEZ, Loreley. Proyecto cultural para la adquisición de competencias laborales en Ingeniería Industrial. Revista de Didáctica Práctica. 2019. 3-8: 35-40.

\footnotetext{
*Correspondencia al Autor (Correo Electrónico: gpejimenezh@yahoo.com)
}

$\dagger$ Investigador contribuyendo como primer autor. 


\section{Introducción}

El Tecnológico Nacional de México (TecNM) comprometido con la educación superior implementa una mejora al modelo por competencias en el 2012, por lo que plantea al Ser Humano como sujeto de aprendizaje, que se forma en una educación integral; el Modelo del Siglo XXI para los Institutos Tecnológicos de México se adecua a las nuevos retos (DGEST, 2012) que se refiere a una serie de planteamientos que constituyen la imagenobjetivo, el deber ser, donde el aprender a aprender, aprender a obtener información, aprender a colaborar, aprender a aplicar conocimientos y aprender a resolver problemas se constituyen en competencias profesionales imprescindibles en una sociedad que demanda profesionistas preparados para solucionar problemas, de acuerdo con Bozu y Canto (2009).

El modelo curricular del TecNM que se ha enriquecido y orientando a una educación de calidad con enfoque en competencias profesionales por personal docente, de investigación y de puestos directivos en las diferentes reuniones nacionales del 2009 a la fecha. Las concepciones de formación profesional, aprendizaje y práctica educativa definidas en el modelo educativo, da un sentido y significado a los contenidos educativos, al quehacer del profesorado y del estudiantado, a las estrategias de aprendizaje y didácticas utilizando las tecnologías de la información y comunicación, así como al diseño e innovación curricular de los planes y programas de estudio que actualmente operan en la formación de profesionales en ingeniería en México. Carranza y González (2016).

La formación integral en la educación se orienta al desarrollo pleno de todas las potencialidades del ser humano; es decir, aunado al cultivo académico, se promueve el crecimiento armónico de la persona desde su riqueza interior, la salud de su cuerpo y su convivencia con los demás. En este propósito, las actividades culturales, artísticas y cívicas son un componente formativo esencial para el desarrollo humano, pues constituyen un eje fundamental para fortalecer el sentido de pertenencia, al tiempo que promueven la articulación y la paz social. TecNM (2014).
En el año 2018 renueva su propuesta con un modelo que refuerza el innovar para innovar, esto introduce a los docentes a generar retos en los procesos de enseñanza, integrar competencias docentes, que permitan al estudiante hacer la apropiación del conocimiento.

Las competencias docente son el resultado de la integración de habilidades, conocimientos y condiciones psicológicas, identificadas por practicantes de la profesión de la enseñanza como esenciales para el dominio del proceso enseñanza-aprendizaje, donde las áreas de docencia, investigación vinculación, gestión, tutoría y formación deben ser sus principales pilares.

Se requiere innovar las estrategias didácticas para el logro del aprendizaje, trabajar por proyectos integrando distintas asignaturas, que permita al estudiante visualizar la utilización de lo estudiado, generando un aprendizaje de la materia, dado que el fenómeno de la reprobación es un elemento de preocupación tanto para docente como para el estudiante y este a su vez es multifactorial, que se originan desde lo familiar, lo económico, los hábitos de estudio, la práctica docente, hasta causas administrativas; es un problema que se agranda con el paso del tiempo, y el cual provoca que los estudiantes abandonen sus estudios generando un nuevo fenómeno, la deserción (Ahumada et al., 2014).

Implementar estrategias en la práctica docente es una opción viable para contribuir al aprendizaje del alumno.

Los proyectos culturales por lo general no se entrelazan con las asignaturas en la generación de competencias laborales, la cultura se visualiza como algo separado de lo académico.

El evento "Porque Calacas, Todos Somos" que se realiza en el municipio de Jiménez, Chih. sirvió como marco para conjuntar las materias de Economía, Metrología y Normalización y Desarrollo Sustentable en una propuesta cultural, que permitiera a los alumnos aplicar conocimientos teóricos, prácticos y actitudinales. 
Dentro de las aulas hay personas que piensan, captan información, la procesan, almacenan y recuperan de forma distinta (Craveri \& Anido, 2009). Los métodos de enseñanza se deben alinear a los estilos de aprendizaje de los estudiantes y de esta manera diseñar nuevas formas de enseñanza. Derivado de ello se realizó una investigación sobre los estilos de aprendizaje de los estudiantes, basados en el test para categorizar los estilos de aprendizaje de acuerdo a Honey Alonso en los estudiantes del ITCdJ, donde se identificaron por carreras los estilos de aprendizaje dominantes.

\section{Desarrollo}

Al implementar estrategias didáctica se debe pensar en los estilos de aprendizaje de los estudiantes con los que se trabaja, debido a que los estilo de aprendizaje es el modo en que una persona aprende, cada persona es un ente individual que refleja diferentes habilidades, debilidades, fortalezas e intereses académicos, es decir la forma preferida del aprendizaje del individuo (Figueroa y Vigliecca, 2006)

Las estrategias de enseñanza incorporadas en un ambiente dentro de salón de clases pueden no ser igual de efectivas en el ambiente de práctica de campo y los estilos de aprendizaje pueden ser parte de un abanico de opciones que visto como competencia puede ser aprovechado mediante el aprendizaje auto-regulado (Gastélum \& Rodríguez, 2012).

Existen distintas teorías de los estilos de aprendizaje, en ellas se observan que son preferencias y disposiciones que tiene un ser humano para aprender. Honey y Mumford asumieron parte de la teoría de Kolb con algunas diferencias, presentan descripciones más detalladas y proponen cuatro estilos de aprendizaje activos, teóricos, reflexivos y pragmáticos, estos estilos fueron modificados por Alonso, Gallego y Honey (1992), Escurra (2011).
Se analizaron los resultados de la investigación sobre estilos de aprendizaje, realizada en el Instituto en el cual se aplicó el cuestionario CHAEA a la muestra dividida por estratos de estudiantes, el cuestionario ha pasado por distintos criterios de confiabilidad y validez por parte de la autora Catalina Alonso en su libro "Estilos de aprendizaje", en lo que respecta a la confiabilidad aplicó la prueba alfa de cronbach en cada uno de los cuatro estilos de aprendizaje. En lo que concierne a la validez realizó los análisis de contenidos, de ítems, y otros tipos de análisis factoriales. Dando por resultado la tabla 1.

\begin{tabular}{|c|r|r|r|r|}
\hline Carrera & Pragmático & Teórico & Reflexivo & Activo \\
\hline $\begin{array}{c}\text { Ingeniero } \\
\text { Industrial }\end{array}$ & 42 & 47 & 10 & 42 \\
\hline
\end{tabular}

Tabla 1 Porcentajes de preferencias alta y muy alta Fuente: elaboración Sánchez et al., (2017)

El estilo teórico es el preferido por los alumnos de Ingeniería Industrial, después de este, el activo supera los porcentajes esperados en los tres últimos rubros. Le siguen el activo y en menor escala el pragmático con un acumulado de $76 \%$ cuando la expectativa es de 70\%. Sánchez et al., (2017).

Uno de los campus pertenecientes al TecNM es el Instituto Tecnológico de Cd. Jiménez, en el cual, con el fin de promover la cultura mexicana implementa un Evento Cultural para finales de Octubre en coordinación con la Presidencia Municipal de Jiménez, la Casa de la Cultura Benjamín Domínguez, el Centro de Educación en Apoyo a la Producción y el Medio Ambiente, A.C. y un grupo de ciudadanos jimenenses. En un esfuerzo por realizar interacciones entre el evento cultural y distintas asignaturas, se propone la intervención didáctica que puedan conjuntar las competencias laborales a desarrollar en los Ingenieros Industriales en formación. Dando a los estudiantes la opción de trabajar en este proyecto o establecer uno distinto según sus creencias y voluntades.

\section{Metodología a desarrollar}

El tipo de investigación es no experimental de alcance descriptivo. Se trata de una intervención didáctica. 


\section{Resultados}

En el semestre de agosto a diciembre del 2018, en las asignaturas de Economía, Metrología y Normalización y Desarrollo Sustentable de la carrera de Ingeniería Industrial, se analizó los estilos de aprendizaje de los estudiantes de Ingeniería Industrial, a partir de ello se les presento a los estudiantes la opción de generar un proyecto que permitiera a los estudiantes adquirir las competencias laborales de cada asignatura $\mathrm{y}$ al mismo tiempo pudiera involucrarse en el proyecto cultural que realiza la Institución o continuar con proyectos independientes. El proyecto fue planteado para la una unidad de cada una de las asignaturas, las cuales tenían sus propios requerimientos.

\section{Competencias de economía:}

- Comprende los fenómenos económicos involucrados en los diferentes procesos involucrados en su área profesional.

- Evaluar y analizar alternativas económicas relacionadas con el consumo y la producción a través de la aplicación de los conceptos, metodología e instrumentos de la teoría microeconómica para el mejoramiento de la productividad de los sistemas productivos.

La unidad es: Teoría de Costos y Producción, que requiere de ubicar las principales teorías de costo y producción, para la toma de decisiones en los procesos acorde a la visión de su empresa; la resolución y análisis de problemas de rendimientos a escala, para lo cual se requiere investigar los conceptos de producto total, promedio y marginal analizando razones para su uso, resolución de problemas de rendimientos de los factores, el representar los diferentes tipos costos en un proceso productivo, asistiendo a una empresa de productos o servicios (TecNM, 2013). En la asignatura Metrología y Normalización, requiere de competencias específicas como: Manejar desde un punto de vista de la metrología y normalización, los métodos y sistemas de medición, para lo cual debe comprender, aplicar y manejar los diferentes instrumentos y equipos de medición en el campo de acción de la metrología; para lo cual debe realizar prácticas de medición a productos utilizando los diferentes instrumentos. (TecNM, 2013).
La materia de desarrollo sustentable se alinea en su visión del entorno económico al hacer uso de los procesos de generación y distribución de la riqueza, así como de los factores y sistemas de producción, la esquematización de costos y la obsolescencia de productos para mejora de la calidad de vida y del ambiente (TecNM , 2014)

La propuesta fue realizar un artículo para el evento cultural "Porque Calacas, Todos somos", donde se realizara una catrina la cual debía tener una proporción entre extremidades y torso, por cada equipo para la asignatura de Meteorología y Normalización, en la cual se debían utilizar los instrumentos de medición para desarrollar las competencias, además debía ser costeada la materia prima, la mano de obra, los costos indirectos de fabricación y realizar una planeación de producción, considerando el cuidado del medio ambiente se propuso utilizaran materiales de reúso, se integraron equipos de cuatro o tres estudiantes, se solicitaron voluntarios para trasladar a los productos a el evento cultural y se sugirió que todos los estudiantes asistieran al evento, como un elemento adicional.

Los estudiantes revisaron y ajustaron las mediciones de las estructuras óseas, así como los elementos de costo de cada uno de sus productos.

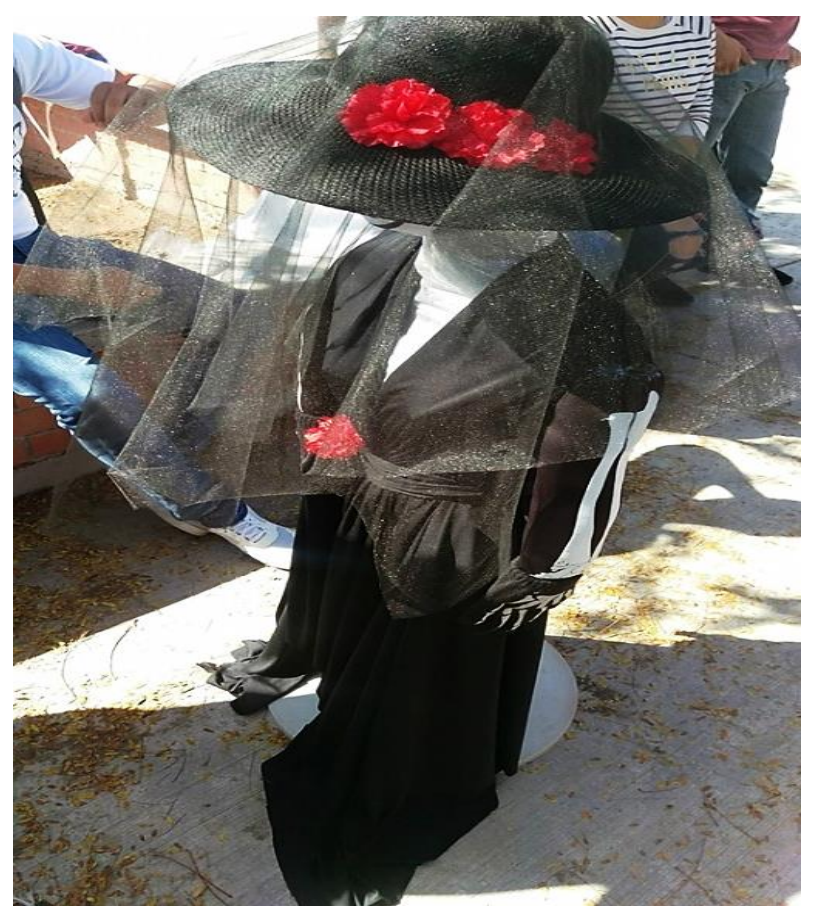

Figura 1 Calaca con base de pedestal de aire acondicionado

Fuente: elaboración propia 


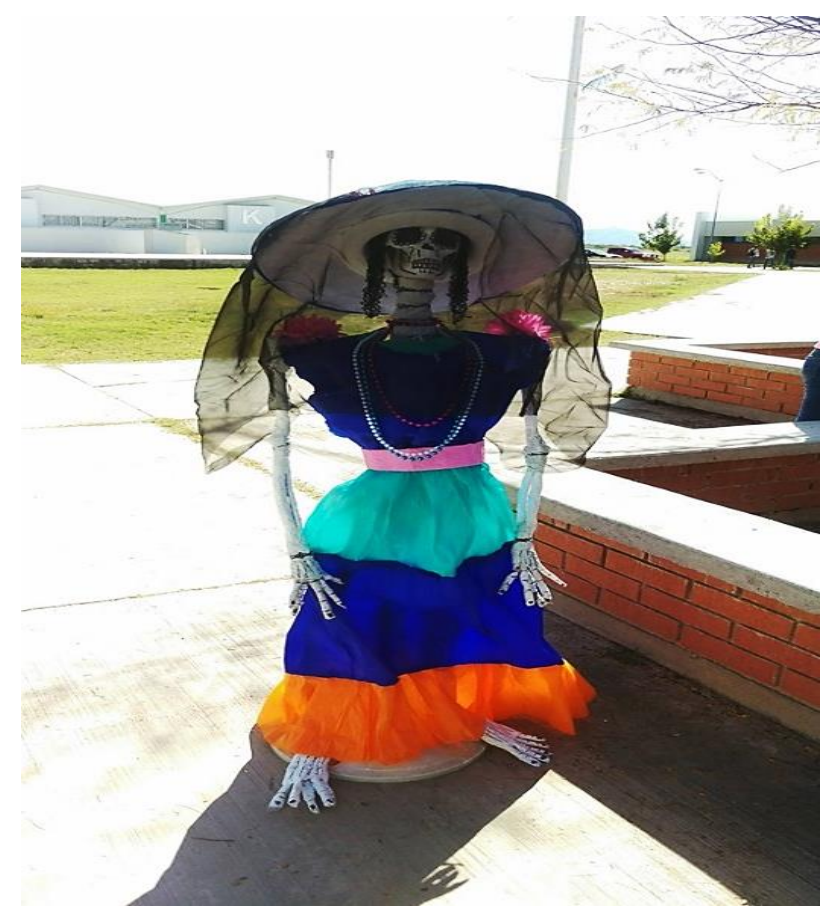

Figura 2 Calaca con base de madera Fuente: elaboración propia

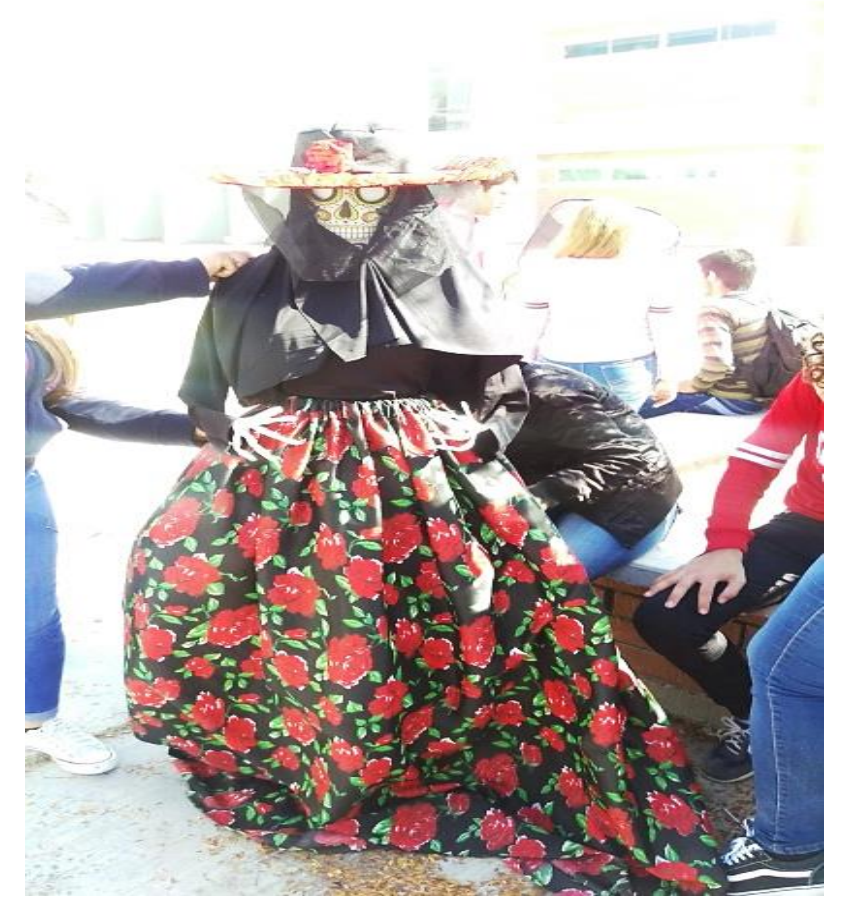

Figura 3 Calaca con base de botes Fuente: elaboración propia

\section{Agradecimiento}

Se agradece al TecNM/ ITCdJ y al CEP, A.C las facilidades en algunos de los materiales de reuso utilizados en las Catrinas.

\section{Conclusiones}

El propiciar ambientes dinámicos donde las practicas didácticos sean versátiles y visibles para incrementar el aprovechamiento de los estudiantes al permitir la interacción de los distintos estilos de aprendizajes, y reducir la reprobación, es un logro, adicionado a ello poder participar en actividades culturales le permite al alumno una formación integral y la generación de competencias. Se logró un promedio de rendimiento de casi $90 \%$ en las unidades implicadas. Utilizar estrategias didácticas que permitan reducir la reprobación, mediante ambientes lúdicos que permitan la comprensión y la apropiación de las competencias laborales, siempre serán bienvenidas.

\section{Referencias}

Ahumada Maldonado Blanca Irene, Jiménez Hidalgo M.A. Guadalupe, Sánchez Lujan Bertha Ivonne y Moreno Kimmy Alina. "Determinantes de reprobación en Ciencias Económico - Administrativas". Memoria de congreso AcademiaJournals Juárez, 2014 ISSN 1948-2353 CD ROM ISSN 1946-5351 Online

Bozu, Z. y Canto P. J. (2009). El profesorado universitario en la sociedad del conocimiento: Competencias profesionales docentes. Revista de Formación e Innovación Educativa Universitaria, 2(2), 87-97. Recuperado de http://refiedu.webs.uvigo.es/Refiedu/Vol2_2/R EFIEDU_2_2_4.pdf

Carranza, A. G., \& González, M. G. A. (2016). Modelo curricular del Tecnológico Nacional de México. Revista electrónica educare, 20(1), 21.

Craveri, A. M., \& Anido, M. (2009). El aprendizaje de la matemática con herramienta computacional en el marco de la teoría de los estilos de aprendizaje. Revista Estilos de Aprendizaje, 102-123.

Dirección General de Educación Superior Tecnológica (DGEST). (2012). Modelo educativo para el siglo XXI: Formación y desarrollo de competencias profesionales. Recuperado de http://www.redalyc.org/pdf/1941/19414301101 0 .pdf 24 
Escurra Mayaute, L. M. (2011). Análisis psicométrico del Cuestionario de Honey y Alonso de Estilos de Aprendizaje (CHAEA) con los modelos de la Teoría Clásica de los Test y de Rasch. Persona, (14), 71-109.

Figueroa, N., \& Vigliecca, M. (2006). Reflexiones sobre nuevos enfoques de enseñanza en ingeniería a partir de experiencias con estilos de aprendizaje. Revista de Informática Educativa y Medios Audiovisuales, 3(7), 32-36.

Gastélum, Y. I. C., \& Rodríguez, A. L. (2012). Aprendizaje auto-regulado como competencia para el aprovechamiento de los estilos de aprendizaje en alumnos de educación superior. Revista de Estilos de Aprendizaje, 5(10).

Sánchez, B. I.; Jiménez, G, Montoya J, Barraza, C. (2016) Las inteligencias múltiples y el rendimiento en matemáticas. Revista de Aplicación Científica y Técnica, 2(3), 27-31

Tecnológico Nacional de México. (2014). Programa Institucional de Innovación y Desarrollo 2013-2018. 50

Tecnológico Nacional de México. (2014). Desarrollo Sustentable. Recuperado el 1 de febrero 2019 en: http://www.itcdjimenez.edu.mx/images/IIND/A C008-Desarrollo-Sustentable.pdf

Tecnológico Nacional de México. (2013). Economía. Recuperado el 1 febrero 2019, en: http://andromeda.itchihuahua.edu.mx/file.php/7 1/Plan_2010_nuevo/Asignaturas\%20Equivalent es/AE018\%20Economia.pdf

Tecnológico Nacional de México. (2013). Metrología y Normalización. Recuperado el 12 de febrero del 2019, en: http://andromeda.itchihuahua.edu.mx/file.php/7 1/Plan_2010_nuevo/Asignaturas\%20Equivalent es/AE048\%20Metrologia\%20y\%20Normalizac ion.pdf 